\title{
CREB and $\mathrm{C} / \mathrm{EBP} \beta$ bind chimeric DNA sites
}

\section{with high affinity}

Jamie R. Flammer, Katerina N. Popova, and Mary Kay H. Pflum

Supplementary Information

Protein expression and purification was assessed with sodium docecyl sulfatepolyacrylamide gel electrophoresis (SDS-PAGE) using a $10 \%$ gel for CREB or a $16 \%$ gel for CEBP $\beta$. Kaleidoscope prestained protein and polypeptide standard (Bio-Rad) were used as a molecular weight markers. Proteins separated by SDS-PAGE were visualized by staining with Colloidal Coomassie Blue (Gradipure, Ltd).
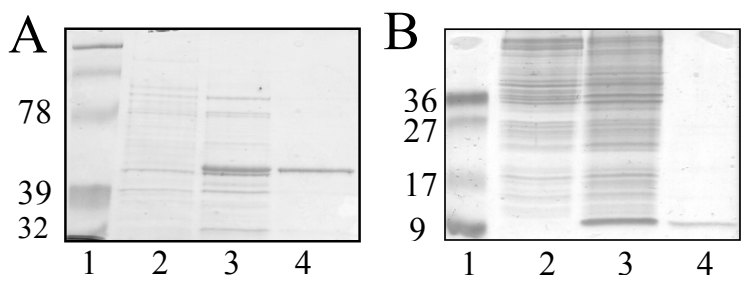

Figure S1. Denaturing SDS-PAGE displaying the purification of CREB (A) and C/EBP $\beta$ (B) using Coomassie staining. Lane 1- Molecular Weight Marker with weight indicated in kDaltons; Lane 2- untreated lysates; Lane 3- lysates after protein expression; Lane 4- purified protein/peptide. 